\title{
Correlation of Levels of $\alpha$-Defensins Determined by HPLC-ESI-MS in Bronchoalveolar Lavage Fluid With the Diagnosis of Pneumonia in Premature Neonates
}

\author{
CHIARA TIRONE, SIMONA BOCCACCI, ROSANNA INZITARI, MILENA TANA, CLAUDIA AURILIA, CHIARA FANALI, \\ TIZIANA CABRAS, IRENE MESSANA, MASSIMO CASTAGNOLA, COSTANTINO ROMAGNOLI, AND GIOVANNI VENTO
}

\begin{abstract}
Department of Pediatrics [C.T., S.B., M.T., C.A., C.R., G.V.], and Institute of Biochemistry and Clinical Biochemistry [R.I., C.F., M.C.], Università Cattolica S. Cuore, 00168 Rome, Italy; Department of Sciences Applied to Biosystems [T.C., I.M.], Cagliari University, 09042
\end{abstract} Monserrato, Italy

\begin{abstract}
The presence of $\alpha$-defensins in bronchoalveolar lavage fluid (BALF) was investigated in a cohort of preterm newborns with gestational age $(\mathrm{GA}) \leq 30 \mathrm{wk}$. Specimens were collected during the first week of life from 24 preterm neonates mechanically ventilated. The studied population was divided into two groups: pneumonia group of nine neonates suffering from pulmonary infection (GA: $26.1 \pm 2.1 \mathrm{wk}$; birth weight: $787.4 \pm 309.9 \mathrm{~g}$ ), with or without associated bloodstream infection, and nonpneumonia group of 15 neonates (GA: $27.7 \pm 2.0 \mathrm{wk}$; birth weight: $1019.0 \pm 319.8 \mathrm{~g}$ ). BALF culture was positive for CONS $(n=5)$, Staphylococcus aureus $(n=1)$, and Candida $\operatorname{spp}(n=3)$. BALF samples were analyzed by HPLC-electrospray Ionization-mass spectrometer. The $\alpha$-defensins 1-4 concentration, absolute and differential white cells count were measured. Relative amounts of $\alpha$-defensins $1-4$ and the absolute number of neutrophils were found significantly higher in the pneumonia group with respect to the nonpneumonia group $(p<0.05)$. Moreover, positive significant correlations between the number of neutrophils and the $\alpha$-defensins $1-3$ levels were observed. In conclusion, our data show that preterm newborns, also at the lower GA, are able to produce $\alpha$-defensins, underlining that their innate defense system is already active before the at-term delivery date. (Pediatr Res 68: 140-144, 2010)
\end{abstract}

A ntimicrobial peptides play an important role in the host innate defense network, even in humans (1). Recent studies demonstrated the capacity of human airways epithelial cells to synthesize antimicrobial peptides and their multifunctional role in the primary immunity (1). Majority of antimicrobial peptides are cationic molecules with hydrophobic and polarized regions, which are essential for their affinity to negatively charged microbial membranes (1-5). According to three-dimensional structure and amino acidic sequence, mammalian antimicrobial peptides can be divided into the following three principal families: cathelicidins, defensins, and histatins $(1,2)$.

Defensins can be divided into $\alpha$ - and $\beta$-subfamilies, on the basis of the different spatial disposition of three disulfide bridges among three couples of cysteine residues. To date, six $\alpha$-defensins have been characterized: the human neutrophil peptides $1-4$, mainly produced by neutrophils, and the human

Received January 5, 2010; accepted April 23, 2010.

Correspondence: Giovanni Vento, M.D., Policlinico A. Gemelli-Università Cattolica S. Cuore, Largo A. Gemelli 8, 00168 Rome, Italy; e-mail: vento@rm.unicatt.it defensins 5-6 expressed by the Paneth's cells (1). They contain 29-35 aminoacids and three disulfides bridges in a $1-6,2-4$, and $3-5$ alignment and reveal a triple stranded $\beta$-sheet structure with a $\beta$-hairpin. Defensins have a broad spectrum of antimicrobial activity against bacteria, fungi, and even some enveloped viruses $(1,5)$. Their arrangement allows them to insert into phospholipid membranes, resulting in the disruption of the normal barrier property of the membrane $(1,5)$. Defensins can also assemble into multimeric pores that permeabilize cellular membranes. Phagocytic cells use defensins during killing and microbic ingestion $(1,5)$. Defensins also lead to cytokines production by epithelium, white cell chemotaxis, wounds repair, and angiogenesis (1).

Several studies suggested that these peptides are involved in the pathogenesis of pulmonary infections (1-7). Pneumonia with or without associated bloodstream infection frequently occurs in preterm neonates requiring ventilatory support (8).

A recent study of Schaller-Bals et al. (9) evaluated, by Immunodot and Western Blotting assay, the presence and concentrations of human $\beta$-defensins $1-2$ and cathelicidin LL-37/hCAP18 in the epithelial lining fluid (ELF) of mechanically ventilated newborns during pulmonary or systemic infection. These authors correlated their concentrations with each other and with inflammatory mediators (TNF- $\alpha$ and IL-8). Differently from murine homologous models, their results show that antimicrobial peptides in newborns are produced independently of the postmenstrual age. They found an increased secretion of these peptides in the ELF during systemic infection with or without pulmonary infection.

It has been reported that bronchoalveolar lavage fluid (BALF) of preterm neonates who need mechanical ventilation is rich in neutrophils (10), and to our knowledge, no specific studies exist about the production of $\alpha$-defensins by neutrophilic cells and on their role in the airways of premature neonates. In a previous work, we evaluated the incidence and etiology of connatal pneumonia and ventilator-associated pneumonia in preterm newborns with birth weight $\leq 1250 \mathrm{~g}$ requiring intubation for at least $12 \mathrm{~h}$. In this study, connatal

Abbreviations: BALF, bronchoalveolar lavage fluid; GA, gestational age; ELF, epithelial lining fluid; ESI, electrospray ionization; MS, mass spectrometer; XIC, eXtracted ion current 
pneumonia and ventilator-associated pneumonia incidence occurred in approximately $8 \%$ and $30 \%$ of patients per year, respectively (11).

In this study, by mass spectrometry, we analyzed whether $\alpha$-defensins $1-4$ are present in the BALF of preterm neonates undergoing mechanical ventilation and the influence of respiratory infections on their concentrations.

\section{METHODS}

Reagents and instruments. All general chemicals and reagents were of analytical grade and were purchased from Farmitalia-Carlo Erba (Milan, Italy), Merck (Damstadt, Germany), and Sigma Chemical Co. Aldrich (St. Louis, MO). The HPLC-electrospray ionization (ESI)-mass spectrometer (MS) apparatus was a surveyor HPLC system (ThermoFinnigan, San Jose, CA) connected by a T splitter to a PDA diode-array detector and to an LCQ Deca XP Plus mass spectrometer. The mass spectrometer was equipped with an electrospray ion source. The chromatographic column was a Vydac (Hesperia, CA) C8 column, with $5 \mu \mathrm{m}$ particle diameter (column dimensions $150 \times 2.1 \mathrm{~mm}$ ). Peptide sequencing was performed with a Procise $610 \mathrm{~A}$ Protein Sequencer (Applied Biosystems, Foster City, CA).

Human participants. The study was performed in the neonatal intensive care unit of the Catholic University of Rome and included 30 premature neonates consecutively admitted between January 2008 and September 2008. Neonates with gestational age (GA) $\leq 30 \mathrm{wk}$ were admitted to the study. They were eligible: (i) when they were born in our hospital; (ii) when endotracheal intubation was required at birth; (iii) when on-going intensive care and mechanical ventilation were required. Newborns with major congenital malformations were excluded from final analysis. Surfactant (a pig-derived, natural surfactant, Curosurf; Chiesi Farmaceutici, Parma, Italy) was administered to all studied newborns, at a dose of $200 \mathrm{mg} / \mathrm{kg}$, as soon as possible after birth, always in the neonatal unit. All the babies were ventilated in elective high-frequency oscillatory ventilation modality with Draeger Baby$\log 8000$ plus (Draeger, Lubeck, Germany), as previously described (12), and received ibuprofen therapy if the ductus arteriosus was hemodynamically significant (13). The study protocol and consent forms were approved by the Ethics Committee of the Department of Pediatrics, and the parents gave their informed consent.

The diagnosis of pneumonia was based on the worsening of respiratory status (increase of fraction of inspired oxygen and/or ventilator setting), with increased amounts of secretions by endotracheal tube, rales, wheezing, persistent chest radiographic abnormalities, and a positive BALF culture (14). Connatal pneumonia (positive BALF culture in the first $24-48 \mathrm{~h}$ of life) and acquired pneumonia (positive BALF culture after $48 \mathrm{~h}$ of life) were distinguished during the study period.

We also evaluated the incidence of some clinical outcomes: sepsis (defined as the presence of clinical signs of infection with a positive blood culture), severe intracranial hemorrhage (grade III or IV), necrotizing enterocolitis, ductus arteriosus surgically ligated, retinopathy of prematurity (stage $>2$ ), bronchopulmonary dysplasia $\left(\mathrm{O}_{2}\right.$-dependence at $36 \mathrm{wk}$ of postmenstrual age), duration of mechanical ventilation, $\mathrm{O}_{2}$ therapy, and survival to discharge.

Sample collection and treatment. BALF samples were obtained in the first $24-48 \mathrm{~h}$ of life, and on day 5-7 of life if the babies were still ventilated, according to a standardized procedure (12). Briefly, two sequential and separate aliquots of $1 \mathrm{~mL} / \mathrm{kg}$ of $0.9 \%$ sodium chloride were instilled through a 6-Fr gauge suction catheter that had been introduced via a slide valve at the endotracheal tube connection and advanced gently until resistance was met, immediately followed by suctioning. After collection, specimens were centrifuged at $1000 \mathrm{~g}$ for $3 \mathrm{~min}$. Cells were suspended in $250 \mu \mathrm{L}$ of $0.9 \%$ sodium chloride and the absolute and differential cell count was obtained by automatic analyzer (Siemens-ADVIA 120, Hematology System, Tarrytown, NY) and by spinning $25 \mu \mathrm{L}$ of suspended cells onto two glass slides using MayGrünwald-Giemsa stain, as previously described (15).

Trifluoroacetic acid $0.2 \%$ solution was immediately added to cell-free supernatants of BALF samples in 1:1 vol/vol ratio, and the solution was centrifuged at $8000 \mathrm{~g}$ for $5 \mathrm{~min}$. The acidic treatment reduced the action of BALF proteases and artifact occurrence. After centrifugation, the acidic supernatants were separated from the precipitate, freeze dried, and resuspended in $300 \mu \mathrm{L}$ of $0.2 \%$ trifluoroacetic acid solution. Hundred microlitres of this soluble fraction from each sample were analyzed by HPLC-ESI-MS.

Moreover, BALF samples were cultured for microbiological analysis including bacteria, Mycoplasma spp, Chlamydia, and fungi to diagnose lung infection.
HPLC-ESI-mass spectrometry analysis. The HPLC-ESI-MS apparatus was connected by a T splitter to a photo diode-array detector and to an LCQ Deca-XP Plus mass spectrometer. The MS apparatus was equipped with an ESI source. The eluents were (A) $0.056 \%$ aqueous TFA and (B) $0.050 \%$ TFA in ACN/water 80:20 vol/vol. The applied gradient was linear from 0 to $55 \%$ of (B) in $40 \mathrm{~min}$, at a flow rate of $0.30 \mathrm{~mL} / \mathrm{min}$. The $\mathrm{T}$ splitter addressed 0.20 $\mathrm{mL} / \mathrm{min}$ toward the diode-array detector and $0.10 \mathrm{~mL} / \mathrm{min}$ toward the ESI source. The diode array detector was set at 214 and $276 \mathrm{~nm}$. Mass spectra were collected every $3 \mathrm{~ms}$ in positive ion mode. MS spray voltage was 4.50 $\mathrm{kV}$ and the capillary temperature was $220^{\circ} \mathrm{C}$.

Deconvolution of average ESI mass spectra was automatically performed either by the software provided with the Deca-XP instrument (Bioworks Browser, San Jose, CA) or by MagTran 1.0 software (16). Experimental mass values were compared with average theoretical mass values using PeptideMass program available at the Swiss-Prot (http://us.expasy.org/tools) data bank: $\alpha$-defensin 1 (Theor Av. Mass $3442.1 \mathrm{D}$, accession code P59665), $\alpha$-defensin 2 (Theor Av. Mass 3371.0 D, accession code P59665/P59666), $\alpha$-defensin 3 (Theor Av. Mass 3486.1 D, accession code P59666), and $\alpha$-defensin 4 (Theor Av. Mass 3709.5 D, accession code P12838).

Quantification of $\alpha$-defensins 1-4. To determine the presence and concentration of the different $\alpha$-defensins, a semiquantitative evaluation was made by performing a multiple eXtracted ion current (XIC) strategy for each $\alpha$-defensin, as previously described (17).

The XIC procedure is based on the extraction of the current associated to either one or multiple selected ions from the total ion current profile. The three most relevant ions of each $\alpha$-defensin were chosen to measure the approximate value of the total ion current associated with each peptide. Under the constant general analytical conditions adopted in this study, the integrated peak area value could be used for a rough estimation of relative changes in protein amount in the different samples (18).

As described in a previous work (19), to normalize the dilution bias resulting from the BALF collection practice and to obtain ELF concentration from the XIC peak area of the different $\alpha$-defensins, the urea method was used (20): ELF concentration $=$ BALF analyte concentration $\times$ (serum urea/BALF urea). Nevertheless, according to the ERS task force recommendations for bronchoalveolar lavage in children (21), which states that results from neonates should be reported as volume instilled, volume recovered, and concentration per millilitre, we presented the data in this way.

Data treatment and statistical analysis. Categorical variables were compared by using a two-tailed Fisher's exact test. Testing for differences of continuous variables between groups was accomplished by Mann-Whitney U test for nonparametric data and $t$ test for parametric data, as appropriate. To evaluate the relationship between $\alpha$-defensins and absolute neutrophils count in BALF samples, linear regression analysis was used. The statistical software used included Instat (GraphPad PRISM Version 3.02). A $p$ value $<0.05$ was considered statistically significant.

\section{RESULTS}

During the study period, 24 neonates were studied: 9 $(37.5 \%)$ developed pneumonia in the first week of life (pneumonia group) and 15 (62.5\%) did not (nonpneumonia group). In the pneumonia group, four neonates had connatal pneumonia (one by Staphylococcus aureus and three by Candida spp) and five neonates had acquired pneumonia with BALF culture positive for coagulase-negative Staphylococci. Three babies with connatal Candida pneumonia were born for mothers with rupture of membranes, with vaginal swabs positive for Candida spp and two of them were born via vaginal delivery. Two neonates suffering from Staphylococcus hemoliticus pneumonia had also a positive blood culture, and a diagnosis of sepsis was made.

Every patient had the first BALF sample on the second day of life and five neonates had a second specimen on the fifth day, because still ventilated and a diagnosis of acquired pneumonia was made thereafter. To avoid a problem of dependence of the data (i.e., 2 samples included for a subgroup of patients), we excluded the first second day BALF sample from the five patients with acquired pneumonia. We finally analyzed 24 BALF samples: 15 samples from neonates 
Table 1. Patients characteristics of the groups analyzed

\begin{tabular}{lcc}
\hline & $\begin{array}{c}\text { Pneumonia group } \\
(N=9)\end{array}$ & $\begin{array}{c}\text { Nonpneumonia group } \\
(N=15)\end{array}$ \\
\hline Gestational age $(\mathrm{yr})$ & $26.1 \pm 2.1$ & $27.7 \pm 2.0$ \\
Birth weight & $787.4 \pm 309.9$ & $1019.0 \pm 319.8$ \\
Male & $2(22)$ & $7(47)$ \\
Appropriate for gestational & $8(89)$ & $13(87)$ \\
$\quad$ age & $5(55)$ & $7(47)$ \\
Antenatal steroids* & $2(22)$ & $1(7)$ \\
Vaginal delivery & $6(67)$ & $4(27)$ \\
Rupture of membranes $\geq 12 \mathrm{~h}$ & \\
Median Apgar score (range) & $3(2-7)$ & $6(3-8)$ \\
$\quad 1$ min $\dagger$ & $7(3-9)$ & $8(5-9)$ \\
5 min &
\end{tabular}

Values expressed as mean $\pm \mathrm{SD}$ and $N(\%)$.

*We included only the newborns receiving a completed course of prenatal betamethasone, defined as two doses administered $>24 \mathrm{~h}$ but not $>7 \mathrm{~d}$ before delivery.

$\dagger p$ value $\leq 0.05$.

Table 2. Clinical and long-term respiratory outcomes; BALF cell count in the studied newborns

\begin{tabular}{lcc}
\hline & $\begin{array}{c}\text { Pneumonia group } \\
(N=9)\end{array}$ & $\begin{array}{c}\text { Nonpneumonia group } \\
(N=15)\end{array}$ \\
\hline $\mathrm{O}_{2}$ dependence at $36 \mathrm{wks} *$ & $6(67)$ & $2(13)$ \\
Bacteriemia or fungemia & $6(67)$ & $5(33)$ \\
Intracranial hemorrage grade & $3(33)$ & $3(20)$ \\
$\quad$ III or IV & & \\
Necrotizing enterocolitis & $2(22)$ & $2(13)$ \\
Ductus arteriosus surgically & $3(33)$ & 0 \\
$\quad$ ligated $\dagger$ & & $7(47)$ \\
Retinopathy of prematurity & $3(33)$ & \\
$\quad$ stage $>2)$ & & $14(93)$ \\
Survival to discharge & $7(78)$ & $210[20-588]$ \\
Mechanical ventilation $(\mathrm{h}) \dagger$ & $476[75-1865]$ & $337[0-1734]$ \\
$\mathrm{O}_{2}$ therapy $(\mathrm{h}) \dagger$ & $875[264-2831]$ & $1.54[0.46-12.19]$ \\
Cells $\left(\times 10^{9} / \mathrm{L}\right) \dagger$ & $5.11[0.69-19.18]$ & $1.32[0.22-10.90]$ \\
Neutrophils $\left(\times 10^{9} / \mathrm{L}\right) \dagger$ & $3.38[0.62-14.26]$ &
\end{tabular}

Values expressed as $N(\%)$ or median [range].

$* p=0.02$.

$\dagger p=0.04$.

of nonpneumonia group (all collected on the 2nd day of life) and 9 samples from neonates of pneumonia group ( 4 collected on the 2 nd day of life-connatal pneumonia, and 5 collected on the 5th day of life-acquired pneumonia). The range of lavage fluid recovery was $75-80 \%$ of the volume instilled $(2 \mathrm{~mL} / \mathrm{kg}$ of $0.9 \%$ sodium chloride) for each BALF sample in all the babies. The characteristics of patients and their major outcomes are summarized in Tables 1 and 2, respectively. No statistically significant differences were found between the two groups of neonates except for the Apgar score at 1 min of life, which was significantly lower in the pneumonia group when compared with the nonpneumonia group $(p<0.05$, Table 1). A higher number of neonates in the pneumonia group were born by vaginal delivery and after rupture of membranes $\geq 12 \mathrm{~h}$ with respect to the nonpneumonia group, but the differences were not statistically significant (Table 1).

The incidence of sepsis, air leaks, necrotizing enterocolitis, and severe intracranial hemorrhage (grade III or IV), was more represented in the pneumonia group with respect to the nonpneumonia group, but the difference was not
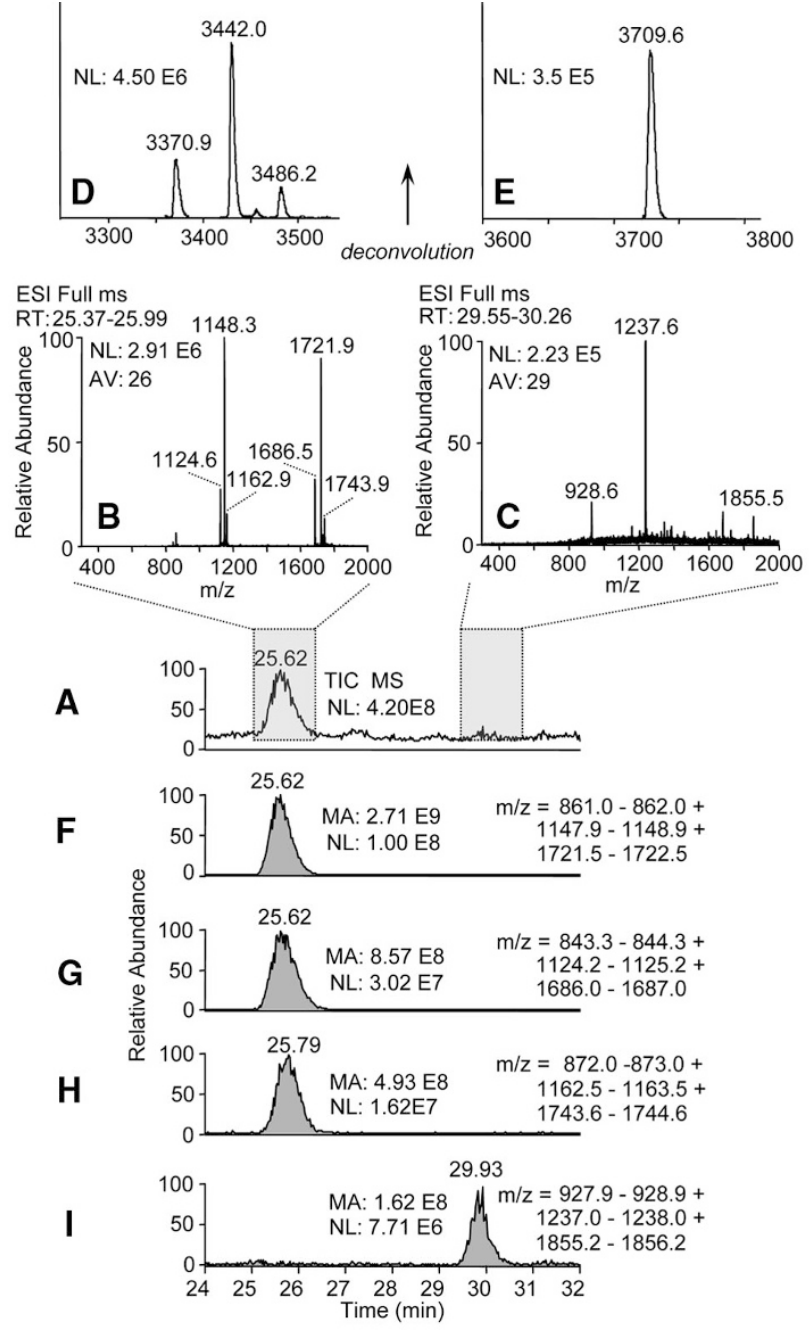

Figure 1. A, Enlargement of the total ion current chromatographic profile of a sample of BALF in the range of 24.0-32.0 min. $B, C$, ESI spectra registered in correspondence of the peaks shown in panels $(F-I)$. Deconvolution of the ESI spectra allowed determining the mass (average) of $\alpha$-defensins 1,2 , and $3(D)$, and $\alpha$-defensin $4(E) . F-I$, XIC peaks of $\alpha$-defensin $1(F), 2(G), 3(H)$, and $4(I)$, respectively. Area of the peaks is proportional to $\alpha$-defensins concentration and was used to evaluate $\alpha$-defensin levels in the different samples. (NL, normalization level; MA, measured area; RT, retention time; $\mathrm{AV}$, number of MS spectra averaged.)

statistically significant (Table 2). The duration of mechanical ventilation and $\mathrm{O}_{2}$ therapy was significantly longer in the pneumonia group $(p<0.05)$ because the incidence of ductus arteriosus surgically ligated and bronchopulmonary dysplasia that were significantly higher in the pneumonia group ( $p<0.05$, Table 2$)$.

Concerning the BALF cytology, both the absolute cell count and the neutrophils absolute number resulted to be significantly higher in the pneumonia group $(p<0.05$, Table 2).

In the HPLC-ESI-MS profile of BALF from premature newborns two peaks belonging to $\alpha$-defensins $1-3$ and $\alpha$-defensin 4 were usually detected (Fig. 1). The MS spectrum registered in the chromatographic range of 25.4-26.0 $\mathrm{min}$ evidenced the presence of three co-eluting peptides with average molecular masses corresponding to $3442.0 \pm 0.4$, $3370.9 \pm 0.4$, and $3486.2 \pm 0.4 \mathrm{D}$, which were attributed to 
$\alpha$-defensins 1,2 , and 3 , respectively. The identification of $\alpha$-defensins 1,2 , and 3 has been previously performed in human salivary samples by using standards (22). The $\alpha$-defensin 4 eluted separately as a distinct peak at 29.5-30.3 min. The experimental average molecular mass value corresponded to $3709.6 \pm 0.4 \mathrm{D}$. The identity of $\alpha$-defensin 4 was confirmed by automated Edman sequencing of the purified peptide. The sequence obtained was VXSXRLVFXRXTELRVGNXLIGGVSF ... in a perfect agreement to that reported in the literature (Swiss Prot code: P12838). $\alpha$-defensins 1 and 2 were detectable in all patients and in all samples. The $\alpha$-defensin 3 and 4 were not detected in only one patient and two patients, respectively.

The levels of each $\alpha$-defensin were significantly higher in the pneumonia group (Fig. 2). A significant positive correlation between the absolute number of neutrophils and the corresponding levels of $\alpha$-defensins 1, 2, and 3 was found (Fig. 3). No differences were observed when correcting data using the urea dilution method, in either the group comparisons or the correlation data. No relationships between $\alpha$-defensins and GA, or between $\alpha$-defensins and bronchopulmonary dysplasia were found (data not shown). An unambiguous
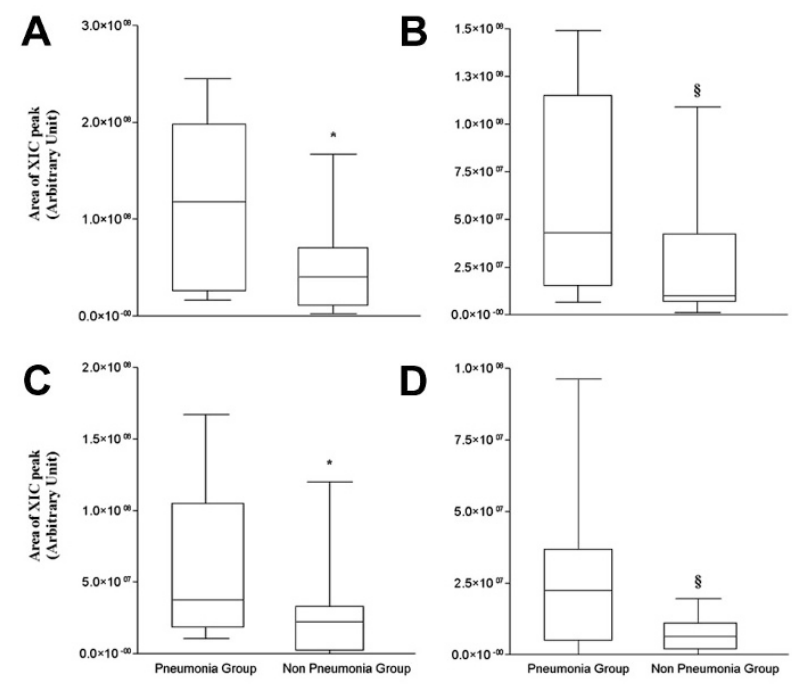

Figure 2. Epithelial lining fluid levels of $\alpha$-defensins $1(A), 2$ (B), 3 (C), and $4(D)$ in the studied newborns. Graphs show data from the 25 th percentile to the 75th percentile, with a line at the median (the 50th percentile) and the highest and lowest values. ${ }^{*} p=0.04 ; \S p=0.03$. temporal pattern of the $\alpha$-defensins $1-4$ in those patients with two collected samples was not found.

\section{DISCUSSION}

The aim of our study was to investigate whether $\alpha$-defensins 1-4 are present in the BALF of preterm neonates undergoing mechanical ventilation and whether respiratory infection affects their levels. To this purpose, the presence and levels of these antimicrobial peptides were evaluated in BALF of preterm neonates by a top-down label free proteomic approach.

Antimicrobial peptides play an important role in innate and adaptive immunity, but until now their role in host defense of newborns is not clear. The $\alpha$-defensins $1-4$ are located in the azurophilic granules of neutrophils. The $\alpha$-defensins $1-3$, which constitute $5 \%$ to $7 \%$ of total protein content of human neutrophils and $30 \%-50 \%$ of the total content of azurophilic granules, represent the most abundant antimicrobial peptides of neutrophils. The $\alpha$-defensin 4 accounts for approximately $1 \%$ of total defensin content (23).

The results of this study show that preterm newborns are able to synthesize $\alpha$-defensins $1-4$ that are present in their ELF. Moreover, the significant positive correlation between the absolute number of neutrophils and the corresponding levels of the $\alpha$-defensins 1-3 suggests that, even in preterm neonates, $\alpha$-defensins might originate prevalently by neutrophilic cells.

Interestingly, we measured significantly higher concentrations of $\alpha$-defensins $1-4$ in the pneumonia than in the nonpneumonia group patients. These data could demonstrate that, at least in part, $\alpha$-defensins $1-4$ contribute also in preterm newborns to the microbicidal and microbiostatic properties of respiratory secretions, and that premature newborns are able to activate an innate immune system of defense. Studies on murine homologs of human antimicrobial peptides indicated that expression of defensins correlates positively with GA (24-26). No specific data exist about a GA dependent expression of $\alpha$-defensins in humans, and our data do not show any correlation between $\alpha$-defensins and GA.

To our knowledge, a proteomic approach (SELDI-TOF MS analysis) for the evaluation of antimicrobial peptides in BALF samples has been only used in one study where concentrations of $\alpha$-defensins 1 and 2 and several forms of S100A proteins were found significantly higher in BALF of patients with
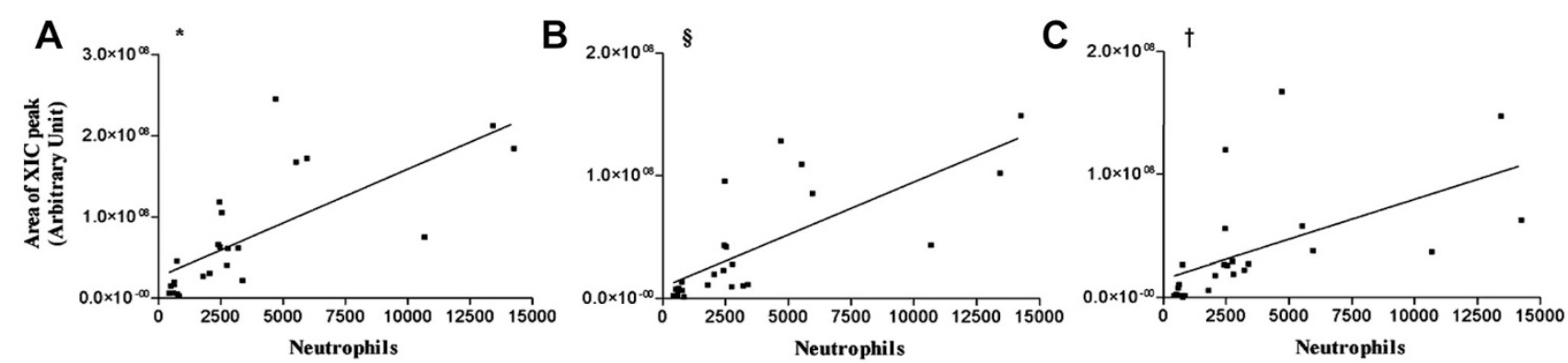

Figure 3. Correlation between levels of $\alpha$-defensins $1(A), 2(B), 3(C)$, and neutrophils in BALF of the studied neonates. The least mean squares regression line is indicated. $* r=0.58, p=0.001 ; \S r=0.52, p=0.0036$; and $\dagger r=0.38, p=0.04$. 
cystic fibrosis than in noncystic fibrosis patients (27). These authors underlined as $\alpha$-defensins are chemotactic for monocytes, stimulate the production of proinflammatory cytokines from monocytes and endothelium, and act on the recruitment, proliferation, and maturation of $\mathrm{T}$ cells. However, the same authors observed how $\alpha$-defensins contribute to tissue damage directly via their cytotoxic effects on cells, and indirectly by competing with neutrophil elastase for the binding of the neutrophil elastase inhibitor $\alpha$-1-antitrypsin. Other studies underline that antimicrobial peptides play a role in the inflammatory lung diseases and how, besides their host defense function during infections, their proinflammatory activity is likely to have negative consequences too $(1,23)$. In this sense, the incidence of bronchopulmonary dysplasia, a multifactorial disease in which cytokines-mediated inflammation play an important role in its pathogenesis (28), was significantly higher in the pneumonia group (with significantly higher $\alpha$-defensins lung levels) than in the nonpneumonia group. Nevertheless, this study was not powered to detect differences in clinical outcomes, such as bronchopulmonary dysplasia, and definitive conclusions cannot be made.

It has been postulated that the effort to develop blood cell-derived proteins and peptides as novel antibiotics could be useful considering the rising prevalence of multidrugresistant microorganisms and the growing population of immunocompromised patients (4). Nevertheless, given the described proinflammatory and antiinflammatory properties of $\alpha$-defensins and the amount of uncertainty regarding their range of actions, it should be cautious at this stage to suggest therapeutic potential of these molecules for preterm infants. We believe that our data may just represent a first step in the knowledge of the biologic functions of $\alpha$-defensins in the innate host defense of the lung against microbial infection in the preterm newborns.

Acknowledgment. We thank the Intensive Care Unit nursing staff for their collaboration for this work.

\section{REFERENCES}

1. Bals R 2000 Epithelial antimicrobial peptides in host defense against infection. Respir Res 1:141-150

2. Yoshio H, Lagercrantz H, Gudmundsson GH, Agerberth B 2004 First line of defense in early human life. Semin Perinatol 28:304-311

3. Ganz T 1999 Defensins and Host defense. Science 286:420-421

4. Levy O 2000 Antimicrobial proteins and peptides of blood: templates for novel antimicrobial agents. Blood 96:2664-2672

5. Ganz T 2004 Antimicrobial polypeptides. J Leukoc Biol 75:34-38

6. Wilson CL, Ouellette AJ, Satchell DP, Ayabe T, López-Boado YS, Stratman JL, Hultgren SJ, Matrisian LM, Parks WC 1999 Regulation of intestinal $\alpha$-defensin activation by metalloproteinase matrilysin in innate host defense. Science 286:113117
7. Koczulla AR, Bals R 2003 Antimicrobial peptides: current status and therapeutic potential. Drugs 63:389-406

8. Cordero L, Ayers LW, Miller RR, Seguin JH, Coley BD 2002 Surveillance of ventilator-associated pneumonia in very-low-birth-weight infants. Am J Infect Control 30:32-39

9. Schaller-Bals S, Schulze A, Bals R 2002 Increased levels of antimicrobial peptides in tracheal aspirates of newborn infants during infection. Am J Respir Crit Care Med 165:992-995

10. Vento G, Romagnoli C, Zecca E, Matassa GP, Tortorolo L, De Carolis MP, d'Onofrio G, Zini G, Tommasi M, Fresu R, Zuppi C 1997 Increased levels of soluble intercellular adhesion molecule-1,neutrophils and elastase in the lung of preterm infants with bronchopulmonary dysplasia. Prenat Neonatal Med 2:348-355

11. Vendettuoli V, De Cunto A, Tana M, Tirone C, Boccacci S, Vento G, Romagnoli C 2007 Pulmonary infections in preterm newborns. Minerva Pediatr 59:97-105

12. Vento G, Matassa PG, Ameglio F, Capoluongo E, Zecca E, Tortorolo L, Martelli M, Romagnoli C 2005 HFOV in premature neonates: effects on pulmonary mechanics and epithelial lining fluid cytokines. A randomized controlled trial. Intensive Care Med 31:463-470

13. Su BH, Watanabe T, Shimizu M, Yanagisawa M 1997 Echocardiographic assessment of patent ductus arteriosus shunt flow pattern in premature infants. Arch Dis Child Fetal Neonatal Ed 77:F36-F40

14. National Nosocomial Infection Survey Manual Available at: http://www.cdc.gov/ ncidod/dhqp/pdf/nhsn/NHSNManual Patient Safety Protocol052407.pdf (Accessed November 16, 2009)

15. Vento G, Matassa PG, Zecca E, Tortorolo L, Martelli M, De Carolis MP, Maggio L, Zini G, D'Onofrio G, Valentini S, Romagnoli C 2004 Effect of dexamethasone on tracheobronchial aspirate fluid cytology and pulmonary mechanics in preterm infants. Pharmacology 71:113-119

16. Zhang Z, Marshall AG 1998 A universal algorithm for fast and automated charge state deconvolution of electrospray mass-to-charge ratio spectra. J Am Soc Mass Spectrom 9:225-233

17. Inzitari R, Vento G, Capoluongo E, Boccacci S, Fanali C, Cabras T, Romagnoli C, Giardina B, Messana I, Castagnola M 2007 Proteomic analysis of salivary acidic proline-rich proteins in human preterm and at-term newborns. J Proteome Res 6:1371-1377

18. Ong SE, Mann M 2005 Mass spectrometry-based proteomics turns quantitative. Nat Chem Biol 1:252-262

19. Vento G, Tirone C, Lulli P, Capoluongo E, Ameglio F, Lozzi S, Cota F, Mosca F, Romagnoli C, Messana I, Castagnola M, Inzitari R 2009 Bronchoalveolar lavage fluid peptidomics suggests a possible matrix metalloproteinase- 3 role in bronchopulmonary dysplasia. Intensive Care Med 35:2115-2124

20. Dargaville PA, South M, Vervaart P, McDougall PN 1999 Validity of markers of dilution in small volume lung lavage. Am J Respir Crit Care Med 160:778-784

21. de Blic J, Midulla F, Barbato A, Clement A, Dab I, Eber E, Green C, Grigg J, Kotecha S, Kurland G, Pohunek P, Ratjen F, Rossi G 2000 Bronchoalveolar lavage in children. ERS Task Force on bronchoalveolar lavage in children. European Respiratory Society. Eur Respir J 15:217-231

22. Pisano E, Cabras T, Montaldo C, Piras V, Inzitari R, Olmi C, Castagnola M, Messana I 2005 Peptides of human gingival crevicular fluid determined by HPLCESI-MS. Eur J Oral Sci 113:462-468

23. Van Wetering S, Sterk PJ, Rabe KF, Hiemstra PS 1999 Defensins: key players or bystanders in infection, injury, and repair in the lung? J Allergy Clin Immunol 104:1131-1138

24. Huttner KM, Kozak CA, Bevins CL 1997 The mouse genome encodes a single homolog of the antimicrobial peptide human beta-defensin 1. FEBS Lett 413:45-49

25. Bals R, Goldman MJ, Wilson JM 1998 Mouse beta-defensin 1 is a salt sensitive antimicrobial peptide present in epithelia of the lung and urogenital tract. Infect Immun 66:1225-1232

26. Morrison GM, Davidson DJ, Kilanowski FM, Borthwick DW, Crook K, Maxwell AI, Govan JR, Dorin JR 1998 Mouse beta defensin-1 is a functional homolog of human $\beta$-defensin-1. Mamm Genome 9:453-457

27. McMorran BJ, Patat SA, Carlin JB, Grimwood K, Jones A, Armstrong DS, Galati JC, Cooper PJ, Byrnes CA, Francis PW, Robertson CF, Hume DA, Borchers CH, Wainwright CE, Wainwright BJ 2007 Novel neutrophil-derived proteins in bronchoalveolar lavage fluid indicate an exaggerated inflammatory response in pediatric cystic fibrosis patients. Clin Chem 53:1782-1791

28. Vento G, Capoluongo E, Matassa PG, Concolino P, Vendettuoli V, Vaccarella C, Frezza S, Zuppi C, Romagnoli C, Ameglio F 2006 Serum levels of seven cytokines in premature ventilated newborns: correlations with old and new forms of bronchopulmonary dysplasia. Intensive Care Med 32:723-730 